\title{
THE ADJOINT OF A BILINEAR OPERATION
}

\section{RICHARD ARENS}

1. Introduction. Let $X, Y, Z$ be normed linear spaces over the field $K$ (complex or real numbers). Denote the space of linear $K$-valued functionals $f$ defined on $X$ and normed in this manner:

$$
\|f\|=\sup _{x \in X}|f(x)|
$$

as usual by $X^{-}$. Let there be defined a bounded bilinear operation on $X \times Y$ with values in $Z$, in symbols:

1.1

$$
m: \quad X \times Y \rightarrow Z \text {, }
$$

$$
m\left(\lambda x_{1}+x_{2}, y\right)=\lambda m\left(x_{1}, y\right)+m\left(x_{2}, y\right),
$$

$$
\begin{aligned}
m\left(x, \lambda y_{1}+y_{2}\right) & =\lambda m\left(x, y_{1}\right)+m\left(x, y_{2}\right), \\
\sup _{\|x\|,\|y\| \leqq 1}\|m(x, y)\| & \leqq M \text { for some } M<\infty .
\end{aligned}
$$

In a previous paper (Arens; these references are to the bibliography of the present paper) there was investigated (in a more general, abstract setting) the process of forming the adjoint operation

$$
m^{*}: Z^{-} \times X \rightarrow Y^{-}
$$

defined, for $f \in Z^{-}, x \in X$, by

$$
m^{*}(f, x)(y)=f(m(x, y))
$$

The simple proof that $m^{*}$ satisfies $1.1,1.2$, and 1.3 with the same value of $M$ is given in (Arens).

This construction can be iterated, and the previous paper was concerned with showing that, in almost every conceivable sense, the operation

$$
m^{* * *}: X^{--} \times Y^{--} \rightarrow Z^{--}
$$

is an extension of $m$. Recall that $X, Y, Z$ are naturally embeddable in $X^{--}, Y^{--}, Z^{--}$resp. Moreover, certain properties, such as associativity, when $m$ has them, are transmitted to $m^{* * *}$ (this makes sense only when $Y=Z=X)$. On the other hand, the transmission of commutativity (which makes sense when $Y=X$ ) was left open, and will be considered in this paper. This question of commutativity can be generalized as follows. If $m$ satisfies $1.1-1.3$, one can define the transposed operation

Received by the editors December 4, 1950. 


$$
m^{t}: \quad Y \times X \rightarrow Z
$$

by $m^{t}(y, x)=m(x, y)$. Since $m^{t * * *}$ is an extension of $m^{t}$, the operation $m^{t * * * t}$ is another extension of $m$. The question of commutativity mentioned above is included in the question: Is $m^{t * * * t}=m^{* * *}$ ? If this is true let us say that $m$ is regular. We shall show below that this is not always true. (In fact, we even show that if $m$ is a commutative associative normed-algebra-operation of multiplication, then $m^{* * *}$ need not be commutative.)

An important special case of an $m$ which satisfies 1.0-1.3 is bounded bilinear functional, where this word is used, as often, to denote that $m(x, y)$ is a number, that is, element of $K$.

When $m$ satisfies $1.0-1.3$ it is possible to characterize $m^{* * *}$ in terms of weak convergence (see $\S 3$ ) as follows: an extension $n$ of $m$ to $X^{--} \times Y^{--}$with values in $Z^{--}$coincides with $m^{* * *}$ if and only if $n(\alpha, \beta)$ is weakly continuous in $\alpha$ when $\beta$ is fixed, and $n(\alpha, \beta)$ is weakly continuous in $\beta$ when $\alpha$ is any fixed element of $X$ (where $X$ is regarded as embedded in $X^{--}$for the moment). Mutatis mutandis, such a characterization holds for $m^{t * * * t}$ also. Thus the regularity of $m$ can be regarded as a certain double weak continuity of $m^{* * *}$ and/or of $m^{t * * * t}$.

Note: In reading this paper, one does well to keep in mind that

$$
\begin{aligned}
m: & X \times Y \rightarrow Z, \\
m^{*}: & Z^{-} \times X \rightarrow Y^{-}, \\
m^{* *}: & Y^{--} \times Z^{-} \rightarrow X^{-}, \\
m^{* * *}: & X^{--} \times Y^{--} \rightarrow Z^{--} .
\end{aligned}
$$

2. Reduction to functionals. Let $Z$ and $W$ be normed linear spaces, and $h$ be a bounded linear operation of $Z$ into $W$, in symbols,

$$
h: Z \rightarrow W \text {. }
$$

As is well known, there is then induced a bounded linear operation $h^{*}$, the adjoint of $h$, of $W^{-}$into $Z^{-}$, where for $k \in W^{-}, h^{*}(k) \in Z^{-}$is defined by $h^{*}(k)(z)=k(h(z))$ for every $z$ in $Z$. One can go further and construct also $h^{* *}: Z^{--} \times W^{--}$, whose behavior as an extension of $h$ is well known.

If $f$ and $g$ are functions where $g$ has domain $X$ and the domain of $f$ includes the range of $g$, one can construct the function with values $f(g(x))$. This function is usually denoted by $f \circ g$.

These conventions are employed in the following proposition.

2.2. TheOREM. Let 1.0-1.3 and 2.1 hold. Then 


$$
h^{* *} \circ m^{* * *}=(h \circ m)^{* * *}
$$

and

$$
h^{* *} \circ m^{t * * * t}=(h \circ m)^{t * * * t} .
$$

These statements make sense because $h \circ m$ evidently satisfies 1.0-1.3-of course, with $W$ taking the place of $Z$.

Proof of 2.2. For $\alpha \in X^{--}, \beta \in Y^{--}$, the left side of 2.21 becomes $h^{* *}\left(m^{* * *}(\alpha, \beta)\right)$, and the right side, $(h \circ m)^{* * *}(\alpha, \beta)$. Evaluated at $l$ in $W^{-}$we get

$$
\begin{aligned}
h^{* *}\left(m^{* * *}(\alpha, \beta)\right)(l) & =m^{* * *}(\alpha, \beta)\left(h^{*}(l)\right) \\
& =\alpha\left(m^{* *}\left(\beta, h^{*}(l)\right)\right)
\end{aligned}
$$

and

$$
(h \circ m)^{* * *}(\alpha, \beta)(l)=\alpha\left((h \circ m)^{* *}(\beta, l)\right)
$$

respectively. Evaluating each of these arguments of $\alpha$ at any $x$ in $X$, we obtain

$$
m^{* *}\left(\beta, h^{*}(l)\right)(x)=\beta\left(m^{*}\left(h^{*}(l), x\right)\right)
$$

and

$$
\left.(h \circ m)^{* *}(\beta, l)(x)=\beta(h \circ m)^{*}(l, x)\right)
$$

respectively. Evaluating each of these arguments of $\beta$ at any $y$ in $Y$ we get

$$
m^{*}\left(h^{*}(l), x\right)(y)=h^{*}(l)(m(x, y))=l(h(m(x, y)))
$$

and

$$
(h \circ m)^{*}(l, x)(y)=l((h \circ m)(x, y))
$$

respectively. Since these are equal, 2.21 is proved. To derive 2.22 from 2.21 replace $m$ by $m^{t}$ and observe that $h \circ m^{t}=(h \circ m)^{t}$.

For the next proposition we have to take a closer look at the "Banach space" $K$, which always denotes the field of scalars, real or complex. $K^{-}$consists of the multiples of $1^{\prime}$ where $1^{\prime}(\lambda)=\lambda, \lambda \in K$; and $K^{--}$consists of the multiplies of $1^{\prime \prime}$ where $1^{\prime \prime}\left(1^{\prime}\right)=1$.

Now, if $m$ satisfies $1.0-1.3$ with $Z=K$, then $m^{* * *}(\alpha, \beta)$ is not numerical-valued, but takes its values in $K^{--}$. A numerical-valued functional can be obtained by setting $n(\alpha, \beta)=m^{* * *}(\alpha, \beta)\left(1^{\prime}\right)$. Then $n(\alpha, \beta) 1^{\prime \prime}=m^{* * *}(\alpha, \beta)$. In this sense, we can speak of $m^{* * *}$ as a functional, an extension of $m$.

2.3. Theorem. Suppose 1.0-1.3 hold. Then $m$ is regular if (and 
only if) $h \circ m$ is a regular functional for every $h$ in $Z^{-}$.

Proof. The "only if" is clear at once, from 2.2. Let us consider the converse, and suppose that $m$ is not regular. There must then exist $\alpha$ in $X^{--}, \beta$ in $Y^{--}$, and $h$ in $Z^{-}$such that

$$
m^{t * * * t}(\alpha, \beta)(h) \neq m^{* * *}(\alpha, \beta)(h) \text {. }
$$

Now it is generally true that for $\gamma \in Z^{--}, \gamma(h)=\left(h^{* *} \circ \gamma\right)\left(1^{\prime}\right)$. In fact, $\left(h^{* *} \circ \gamma\right)\left(1^{\prime}\right)=h^{* *}\left(\gamma\left(1^{\prime}\right)\right)=\gamma\left(h^{*}(1)\right)$, and $h^{*}(1)(z)=1^{\prime}(h(z))$ $=h(z)$ so that $h^{*}\left(1^{\prime}\right)=h$. We conclude that

$$
h^{* *} \circ m^{t * * * t}(\alpha, \beta) \neq h^{* *} \circ m^{* * *}(\alpha, \beta) \text {. }
$$

The assumption that $h \circ m$ is regular and the above now contradict 2.21 and 2.22. Thus 2.3 is proved.

3. Characterization of $m^{* * *}$ by weak convergence. If $m$ satisfies $1.0-1.3$, of course $m^{* * *}$ is continuous in the norm topology. In this section we make some statements about its continuity relative to "weak" topologies.

Let $X$ be any topological linear space, and let $X^{-}$be the space of linear continuous functionals. Then the $p$ topology in $X^{-}$is that topology in which a directed set $f_{\mu}$ converges to a limit $f$ if and only if the numbers $f_{\mu}(x)$ converge to $f(x)$ for every $x$ in $X$. Besides the $p$ topology, when $X$ is a normed linear space, there is the "bounded" $p$ topology (call it the $\bar{p}$ topology), in which $f_{\mu}$ converges to $f$ if, first, the $\left\{f_{\mu}\right\}$ constitute a bounded set and, second, they converge to $f$ in the $p$ topology (Dieudonné). Now assume $\phi$ is a linear mapping of $X^{-}$ to $Y^{-}$continuous when the $p$ topology is used in both $X^{-}$and $Y^{-}$. We then say $\phi$ is $p-p$ continuous. An analogous definition defines $\bar{p}-\bar{p}$ continuity. Of course,

3.1. $p-p$ continuity implies $\bar{p}-\bar{p}$ continuity for bounded linear operations.

When $X$ is a normed linear space, and $x \in X$, there is an element $\alpha$ in $X^{--}$such that $f(x)=\alpha(f)$ for every $f$ in $X^{-}$. This $\alpha$ we shall consistently denote by $\dot{x}$.

3.2. THEOREM. Let $m$ satisfy 1.0-1.3. Then

3.21. for fixed $\beta$, resp. $y, m^{* * *}(\alpha, \beta)$ resp. $m^{t * * * t}(\alpha, \dot{y})$ is $p-p$ and also $\bar{p}-\bar{p}$ continuous in $\alpha$; and

3.22. for fixed $\alpha$, resp. $x, m^{t * * * t}(\alpha, \beta)$ resp. $m^{* * *}(\dot{x}, \beta)$ is $p-p$ and also $\bar{p}-\bar{p}$ continuous in $\beta$.

Proof. Consider first 3.21. Let $\alpha_{\mu} \rightarrow 0$ in the $p$ topology of $X^{--}$. Then, for $h$ in $Z^{--}$, 


$$
m^{* * *}\left(\alpha_{\mu}, \beta\right)(h)=\alpha_{\mu}\left(m^{* *}(\beta, h)\right) \rightarrow 0
$$

furthermore,

$$
\begin{aligned}
m^{t * * * t}\left(\alpha_{\mu}, \dot{y}\right)(h) & =m^{t * * *}\left(\dot{y}, \alpha_{\mu}\right)(h) \\
& =\dot{y}\left(m^{t * *}\left(\alpha_{\mu}, h\right)\right) \\
& =m^{t * *}\left(\alpha_{\mu}, h\right)(y) \\
& =\alpha_{\mu}\left(m^{t *}(h, y)\right) \rightarrow 0
\end{aligned}
$$

This, together with 3.1 , proves 3.21 . The proof of 3.22 is similar.

This result characterizes $m^{* * *}$ and $m^{t * * * t}$. The next proposition characterizes those $m$ which are regular, that is, for which $m^{* * *}$ $=m^{t * * * t}$. By an extension $n$ of $m$ we shall mean a bounded bilinear operation satisfying $1.0-1.3$ with $X, Y, Z$ replaced by $X^{--}, Y^{--}$, $Z^{--}$respectively, such that $n(\dot{x}, \dot{y})=m(x, y)$.

3.3. Theorem. Let $m$ satisfy 1.0-1.3. Then the following seven conditions are equivalent.

3.31. $m$ is regular;

3.32. $m^{t}$ is regular;

3.33. $m^{* * *}(\alpha, \beta)$ is $p-p$ continuous in $\beta$ for each $\alpha$;

3.34. $m^{* * *}(\alpha, \beta)$ is $\bar{p}-\bar{p}$ continuous in $\beta$ for each $\alpha$;

3.35. $m^{t * * * t}(\alpha, \beta)$ is $p$-p continuous in $\alpha$ for each $\beta$;

3.36. $m$ has an extension $n$ such that $n(\alpha, \beta)$ is $p$-p continuous in $\alpha$ for each $\beta$ and in $\beta$ for each $\alpha$;

3.37. $m$ has an extension $n$ such that $n(\alpha, \beta)$ is $\bar{p}-\bar{p}$ continuous in $\alpha$ for each $\beta$ and in $\beta$ for each $\alpha$.

The proof of 3.3 is to be based on the validity of the implications $3.31 \leftrightarrow 3.32,3.31 \rightarrow 3.33 \rightarrow 3.34 \rightarrow 3.31 \rightarrow 3.35 \rightarrow 3.36 \rightarrow 3.37 \rightarrow 3.31$.

For $3.31 \leftrightarrow 3.32$, these are obviously equivalent.

For $3.31 \rightarrow 3.33$, this follows from 3.2.

For $3.33 \rightarrow 3.34$, this follows from 3.1.

For $3.34 \rightarrow 3.31$, first observe that for any $\alpha$,

$$
m^{t * * * t}(\alpha, \dot{y})=m^{* * *}(\alpha, \dot{y}) \text {. }
$$

In fact, evaluate both sides at $h \in Z^{--}$. There will result $m^{t * * * t}(\alpha, \dot{y})(h)=m^{t * * *}(\dot{y}, \alpha)(h)=m^{t * *}(\alpha, h)(y)=\alpha\left(m^{t *}(h, y)\right)$ and $\alpha\left(m^{* *}(\dot{y}, h)\right)$, respectively. Evaluating each of the arguments here at any $x$ in $X$ yields, after simplification, $h(m(x, y))$ in both cases. Next we recall that for each $\beta \in Y^{--}$one can construct a directed set $y_{\mu} \in Y$ such that $\dot{y}_{\mu} \rightarrow \beta$ in the $\bar{p}$ topology of $Y^{--}$(see Helly's theorem, in the form presented by Kakutani). We now replace $y$ in 3.4 by $y_{\mu}$ and take limits, using 3.34. This yields 3.31 . 
For $3.31 \rightarrow 3.35$, this is just like $3.31 \rightarrow 3.33$. Notice that we have omitted the "transposed" form of $3.33 \rightarrow 3.34 \rightarrow 3.31$, for brevity.

For $3.35 \rightarrow 3.36, m^{t * * * t}$ provides the $n$ at once.

For $3.36 \rightarrow 3.37$, this follows from 3.1 .

For $3.37 \rightarrow 3.31$, by the use of Helly's theorem and the continuity properties of $m^{* * *}$ and $m^{t * * * t}$ set forth in 3.2 , one can prove that each of the latter extensions coincide with $n$. We omit the details, since they closely resemble those provided for $3.34 \rightarrow 3.31$.

Thus 3.3 is proved. We shall utilize later the implication $3.34 \rightarrow 3.31$.

At this point we would like to point out the interesting special case of a symmetric $m$. If $X=Y$ it may happen that $m=m^{t}$, in which case $m$ is called symmetric. A symmetric $m$ is regular if and only if $m^{* * *}=m^{t * * * t}=m^{* * * t}$, that is, if and only if $m^{* * *}$ is symmetric. A symmetric $m$ is regular if and only if it has any extension $n \bar{p}-\bar{p}$ continuous in either variable, of course.

4. A condition implying regularity. A weakly compact subset of a normed linear space $X$ is a set which is compact in the weak topology of $X$ induced by the functionals in $X^{-}$(see Alaoglu).

We here study the implications of the following condition, wherein $m$ is supposed to satisfy $1.0-1.3$.

4.1. For every positive $\epsilon$ there exists a weakly compact subset $\Gamma$ contained in the unit ball $U$ of $X$ such that for every $x$ in $U$ there is an $x_{\epsilon}$ in $\Gamma$ such that for every $y$ in $Y$

$$
\left\|m\left(x-x_{\epsilon}, y\right)\right\| \leqq \epsilon\|y\| \text {. }
$$

This condition is a generalization of "complete continuity," for bilinear functionals, which requires the existence of a finite set $\Gamma$ with the properties mentioned. However, 4.1 is automatically satisfied when $X$ is a Hilbert space, or indeed any reflexive space, for by a theorem of Alaoglu and Bourbaki (Alaoglu), $U$ is then weakly compact and may be taken for $\Gamma$ with every $\epsilon$.

4.2. LEMma. If 4.11 holds for every $y$, then for every $\beta \in Y^{--}$such that $\|\beta\| \leqq 1$,

$$
\left\|m^{* * *}\left(\dot{x}-\dot{x}_{\epsilon}, \beta\right)\right\| \leqq \epsilon\|\beta\| .
$$

We leave the proof to the reader.

The following is the main theorem of this section.

4.3. Theorem. Suppose $m$ satisfies 1.0-1.3 and also 4.1. Then $m$ is regular.

Proof. We prepare to apply 3.3 in the form $3.34 \rightarrow 3.31$. It will suffice to show that $m^{* * *}(\alpha, \beta)$ is $\bar{\phi}-\bar{p}$ continuous at $\beta=0$, for any 
fixed $\alpha$ with $\|\alpha\| \leqq 1$. Since $m^{* * *}$ is homogeneous it will suffice to show that for each $\epsilon>0$ and $h \in Z^{-}$we can find a $p$-neighborhood $V$ of 0 in $Y^{--}$such that $\beta \in V$ implies

$$
\left|m^{* * *}(\alpha, \beta)(h)\right|<3 \epsilon .
$$

To the given $\epsilon$, apply 4.1 obtaining a $\Gamma$ as set forth.

We now approximate to $\alpha$, by elements of $X$. For every finite subset $\phi=\left\{f_{1}, \cdots, f_{n}\right\}$ of $X^{-}$there exists an $x_{\phi} \in X$ such that $\left\|x_{\phi}\right\| \leqq 1$ and

$$
\alpha\left(f_{i}\right)=f_{i}\left(x_{\phi}\right) \quad(i=1,2, \cdots, n),
$$

by Helly's theorem as formulated by Kakutani, for example. For each $x_{\phi}$ obtain an $x_{\phi \epsilon} \in \Gamma$ such that 4.21 will hold with $\|\beta\| \leqq 1$, that is,

$$
\left\|m^{* * *}\left(\dot{x}_{\phi}-\dot{x}_{\phi \epsilon}, \beta\right)\right\| \leqq \epsilon .
$$

These $x_{\phi \epsilon}$ regarded as a set directed by the $\phi$ have at least one cluster point $x$ in $X$. Let $V$ be the neighborhood of 0 in $Y^{--}$under the $\bar{P}$ topology, defined by

$$
\left|\beta\left(m^{*}(h, x)\right)\right|<\epsilon,
$$

$$
\|\beta\| \leqq 1
$$

Suppose $\beta \in V$. Select any $\phi$ such that

$$
\left|m^{* *}(\beta, h)\left(x_{\phi \epsilon}-x\right)\right|<\epsilon
$$

and

$$
m^{* *}(\beta, h) \in \phi .
$$

This is possible because the $\phi$ satisfying 4.35 are a cofinal set and those satisfying 4.36 are, of course, a final set. From 4.36 we obtain $\left(\alpha-\dot{x}_{\phi}\right)\left(m^{* *}(\beta, h)\right)=0$ and from 4.33 we get $\left|\left(\dot{x}_{\phi}-\dot{x}_{\phi \epsilon}\right)\left(m^{* *}(\beta, h)\right)\right|$ $<\epsilon$. Applying these two relations as well as $4.34,4.35$ to the equation

$$
\begin{aligned}
m^{* * *}(\alpha, \beta)(h)= & \left(\alpha-\dot{x}_{\phi}\right)\left(m^{* *}(\beta, h)\right)+\left(\dot{x}_{\phi}-\dot{x}_{\phi \epsilon}\right)\left(m^{* *}(\beta, h)\right) \\
& +m^{* *}(\beta, h)\left(x_{\phi \epsilon}-x\right)+\beta\left(m^{*}(h, x)\right),
\end{aligned}
$$

we obtain 4.31 . Thus 4.3 is proved.

In 4.3, condition 4.1 may be replaced by another in which $X$ and $Y$ are interchanged. One then interchanges $Y$ and $X$, applies 4.3, and shows $m^{t}$ to be regular. In particular, it follows that if $X$ or $Y$ is reflexive, then $m$ is regular. This fact can also be deduced directly from the definition of $m^{* * *}$.

I have not been able to find necessary conditions along the lines of 4.3 involving compact sets. At present we shall have to content ourselves with counterexamples given in the next section. The simplest nonreflexive Banach space that one might approach in 
order to construct an $m$ which is not regular is undoubtedly $\left(c_{0}\right)$ in the notation of Banach, for then the conjugate spaces are successively $(l)$ and $(m)$ (see Banach) and thus readily available. However, it so happens that no counterexample with $X=Y=\left(c_{0}\right)$ is possible, since it is proved by Littlewood that every bilinear functional on $\left(c_{0}\right) \times\left(c_{0}\right)$ is completely continuous. Thus we obtain the following result.

4.4. ThEOREM. If $m$ satisfies $1.0-1.3$ where $X=Y=\left(c_{0}\right)$, then $m$ is regular.

To prove this theorem, one must verify that every such bilinear form belongs to the class considered by Littlewood, but this is easy to do since $\left(c_{0}\right)$ has a basis.

5. Bilinear forms in $(l)$. As in Banach's notation, let $(l)$ be the space of absolutely summable sequences $\left(\xi_{1}, \xi_{2}, \cdots, \xi_{n}, \cdots\right)$ with $\sum\left|\xi_{n}\right|$ as the norm. Let $x_{n}$ denote that element of $(l)$ whose $k$ th term is 0 unless $k=n$, when it is 1 . Let $m:(l) \times(l) \rightarrow K$ satisfy $1.1-1.3$. In this section, $K$ shall be the real field, for definiteness and simplicity. By setting $\mu_{i j}=m\left(x_{i}, x_{j}\right)$ we obtain a uniformly bounded array of numbers

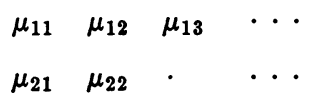

$\mu_{31}$

$$
\left(\left|\mu_{i j}\right| \leqq M\right)
$$

such that

$$
m(x, y)=\sum \mu_{i j} \xi_{i} \eta_{j}
$$

where $x=\left(\xi_{1}, \xi_{2} \cdots\right), y=\left(\eta_{1}, \eta_{2}, \cdots\right)$, elements of $(l)$. Conversely, given $5.1,5.11$ defines an $m$ of the sort considered.

To examine $m^{*}: K^{-} \times(l) \rightarrow(l)^{-}$we first observe that $(l)^{-}=(m)$, and then recall that we have been using $1^{\prime}$ to denote the element of $K^{-}$such that $1^{\prime}(\lambda)=\lambda$ for $\lambda \in K$. It is easy to see that

$$
m^{*}\left(1^{\prime}, x\right)=\left(\sum \mu_{i 1} \xi_{i}, \sum \mu_{i 2} \xi_{i}, \cdots\right) \in(m) .
$$

Our next step is the following.

5.3. Lemma. Let $\alpha \in(m)^{-}$. Then $m^{* *}\left(\alpha, 1^{\prime}\right) \in(m)$ and its nth component is (the value of) $\alpha$ applied to the nth row of 5.1 .

Proof. The $n$th component is $m^{* *}\left(\alpha, 1^{\prime}\right)\left(x_{n}\right)=\alpha\left(m^{*}\left(1^{\prime}, x_{n}\right)\right)$ $=\alpha\left(\mu_{n 1}, \mu_{n 2}, \mu_{n 3}, \cdots\right)$, by 5.2 . 
As we pointed out before, $m^{* * *}(\alpha, \beta)$ is not a number, but a multiple $\mu 1^{\prime \prime}$ of $1^{\prime \prime}$ where $1^{\prime \prime}\left(1^{\prime}\right)=1$ and $1^{\prime}(\lambda)=\lambda$ for $\lambda \in K$. However, we may identify it with $\mu$. Hence our final result may be put as follows.

5.4. Theorem. Let $\alpha, \beta \in(m)$-. Then $m^{* * *}(\beta, \alpha)$ is calculated as follows. Apply $\alpha$ to each row of 5.1, obtaining a sequence of numbers which belongs to $(m)$, and then apply $\beta$ to this sequence. The result is $m^{* * *}(\beta, \alpha)$. On the other hand, if $\beta$ is applied to each column of 5.1 and then $\alpha$ is applied to the sequence thus obtained, the result is $m^{t * * * t}$.

PROOF. $m^{* * *}(\beta, \alpha)\left(1^{\prime}\right)=\beta\left(m^{* *}\left(\alpha, 1^{\prime}\right)\right)$-the rest is obvious.

We are now ready to consider examples of $m$ which are not regular. Consider the $m$ defined by the array

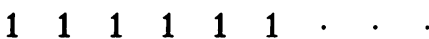

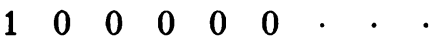

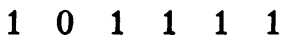

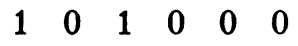

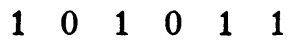

$\begin{array}{llllll}1 & 0 & 1 & 0 & 1 & 0\end{array}$

on which the diagonal elements alternate between 1 and 0 , each nondiagonal element is equal to the diagonal element above or to the left of itself. This (5.5) is evidently a symmetric $m$.

Recall that $(m)$ is a normed linear algebra, with the addition already considered and the multiplication defined by

$$
\left(u_{1}, u_{2}, \cdots\right)\left(v_{1}, v_{2}, \cdots\right)=\left(u_{1} v_{1}, u_{2} v_{2}, \cdots\right) .
$$

The class of elements of $(m)$ which have only a finite number of nonzero components clearly form an ideal $J_{\infty}$. Let $M_{1}$ be a maximal ideal of $(m)$ which contains $J_{\infty}$ as well as the element $(0,1,0,1,0, \cdots)$. Let $M_{2}$ be a maximal ideal of $(m)$ containing $J_{\infty}$ and $(1,0,1,0, \cdots)$. These ideals exist by Zorn's lemma since $J_{\infty}$ plus $(0,1,0,1, \cdots)$ resp. plus $(1,0,1,0, \cdots)$ do not generate all of $(m)$. Since $(m)$ can be represented as the algebra of all continuous real-valued functions on a compact Hausdorff space (in fact, on the Cech compactification of the integers: see for example Nakamura and Kakutani), $M_{1}$ and 
$M_{2}$ are kernels of certain real-valued linear algebra-homomorphisms of $(m)$. Call these resp. $\alpha$ and $\beta$. Now the values of $\alpha$ (and indeed also the values of $\beta)$ for the rows of 5.5 are in turn $(1,0,1,0,1,0, \ldots)$ (and the same thing is true for the columns), since each row (or column) differs from $(0,0,0, \ldots)$ or $(1,1,1,1, \cdots)$ by only finitely many components. Hence $m^{* * *}(\beta, \alpha)=\beta(1,0,1,0, \cdots)=0$. On the other hand, $m^{t * * * t}(\beta, \alpha)=\alpha(1,0,1,0, \cdots)$. This number is not 0 but rather 1 since

$$
(1,0,1,0, \cdots)+(0,1,0,1, \cdots)=(1,1,1,1, \cdots)
$$

and so

$$
\alpha(1,0,1,0, \cdots)+\alpha(0,1,0, \cdots)=1,
$$

and we provided that the second term should vanish.

By a consideration of the bounded linear functionals on $(m)$ as represented in the paper of Kakutani and Nakamura it is not hard to see that an $m$ as given by 5.1 is regular if (and of course only if) $m^{* * *}(\beta, \alpha)=m^{t * * * t}(\beta, \alpha)$ for merely those $\alpha, \beta$ which are real-valued normed linear algebra homomorphisms of $(m)$ which vanish on the ideal $J_{\infty}$, that is, correspond to points of $\omega^{-}-\omega$ where $\omega$ is the set of integers and $\omega^{-}$is its Cech compactification using the discrete topology.

The preceding $m$ can be used to make $(l)$ into an associative commutative normed linear algebra $A$, by setting

$$
M(x, y)=(m(x, y), 0,0, \cdots) .
$$

Then $m=h \circ M$ where $h\left(\zeta_{1}, \zeta_{2}, \cdots\right)=\zeta_{1}$. Therefore $M$ is not regular, by 2.2 , and hence not commutative, by the remark following 3.3 .

\section{BIBLIOGRAPHY}

L. Alaoglu, Weak topologies of normed linear spaces, Ann. of Math. (2) vol. 41 (1940) pp. 252-267.

R. Arens, Operations induced in function classes, Monatshefte für Mathematik vol. 55 (1951).

S. Banach, Thêorie des opérations linéaires, Warsaw, 1932.

J. Dieudonne, Natural homomorphisms in Banach spaces, Proceedings of the American Mathematical Society vol. 1 (1950) pp. 54-59.

S. Kakutani, Weak topology and regularity of Banach spaces, Proc. Imp. Acad. Tokyo vol. 15 (1939) pp. 169-173.

S. Kakutani and M. Nakamura, Banach limits and the Čech compactification of a countable discrete set, Proc. Imp. Acad. Tokyo vol. 19 (1943) pp. 224-229.

J. A. Littlewood, On bounded bilinear forms, Quarterly Journal of Mathematics vol. 1 (1930) pp. 164-174.

University of California, Los Angeles 